\title{
¿Cuáles son las barreras de acceso a la atención odontológica de las mujeres embarazadas? Una revisión de la literatura
}

\author{
Cantarutti ${ }^{1}$, Veliz $C^{1}$, Pardo A ${ }^{1}$, Ortúzar I ${ }^{1}$, Jordan V ${ }^{1}$, Moneva $\mathrm{M}^{2}$, Madariaga $\mathrm{P}^{3}$
}

Resumen: Introducción: Las enfermedades bucales son altamente prevalentes a nivel mundial; una de las estrategias utilizadas para mejorar el estado de salud bucal de la población es la atención odontológica de la embarazada. En Chile esta atención está garantizada, sin embargo, la cobertura de las atenciones no es la esperada, sólo el 31\% de las mujeres embarazadas accede a la atención odontológica y un $14 \%$ abandona el tratamiento antes de terminarlo.

Métodos: El objetivo de este artículo es revisar la evidencia internacional y nacional respecto a las barreras que impiden a las mujeres embarazadas acceder al tratamiento odontológico.

Resultados: Se describen diferentes barreras para la atención odontológica de la mujer embarazada, las que se pueden clasificar en las relacionadas con la organización del sistema de salud (costo de la atención, acceso a los prestadores y las características de los proveedores de la atención de salud) y las barreras personales (creencias de las pacientes, percepción de necesidad de tratamiento, miedo a la atención y valoración de la salud bucal).

Conclusión: Existe evidencia internacional sobre cuáles son las barreras para el acceso a la atención odontológica de la mujer embarazada, entre ellas, destacan las barreras de acceso, como el costo de atención, sin embargo, a nivel nacional, esa barrera está soslayada, ya que el acceso y la protección financiera están garantizadas gracias a la cobertura GES. Por esta razón son necesarios estudios nacionales que indaguen sobre el fenómeno que impide que las embarazadas chilenas accedan al tratamiento odontológico.

Palabras clave: embarazo; cuidado dental; acceso a los servicios de salud; barreras para la atención; cobertura atención; GES.

Abstract: Introduction: Oral diseases are highly worldwide prevalent; one of the strategies used to improve oral health status of the population is the specific pregnant women dental care program. In Chile this care is guaranteed by law, however, the dental attention coverage is not as well as expected, only $31 \%$ of pregnant women have access to dental care and $14 \%$ of them, leave treatment before ending.

Methods: The aim of this article is to review international and national evidence regarding the barriers that prevent pregnant women from accessing to dental treatment.

Results: Different barriers are described. They can be classified in those related to the organization of the oral health system (care cost access to providers, care health providers characteristics) and personal barriers (patient's beliefs, perceived needs for treatment, fear and oral health assessment).

Conclusion: There is international evidence about the barriers to access to pregnant women, dental care including barriers access such the care cost; however, at the national level, this barrier is overlooked since access and the financial protection is guaranteed by GES coverage. More studies are needed to investigate the phenomenon that avoids Chilean pregnant women from accessing to dental treatment.

Keywords: Pregnancy; Dental care; Access to health services; Barriers to health care; Coverage; GES.

Fecha de envío: 25 de mayo de 2017 - Fecha de aceptación: 9 de agosto de 2017

1) Escuela de Odontología, Facultad de Medicina, Pontificia Universidad Católica de Chile

2) CESFAM Flor Fernández

3) Red de Salud UC-Christus

Autor de correspondencia: ccantarutti@uc.cl 


\section{Introducción}

A nivel mundial, la prevalencia de las enfermedades bucodentales es cercana al 100\% en la población adulta (OMS, 2012). En Chile la situación es similar, casi el 100\% de los adultos presentan historia de caries (MINSAL, 2010).

Las mujeres chilenas en edad reproductiva presentan una alta prevalencia de caries. El 57,3\% de las mujeres de 17 a 24 años y el $73,5 \%$ entre 25 y 44 años presentan caries (MINSAL, 2003). Respecto a la pérdida de dientes, el 61,9\% de las mujeres entre 17 a 24 años presenta su dentición completa, porcentaje que disminuye a un $31,9 \%$ en el tramo de edad entre los 25 y 44 años (MINSAL, 2003).

La evidencia internacional revela que el estado de salud bucal y el acceso a la atención muestran inequidades. Se observa que los sectores de menor NSE y NEDU presentan un peor estado de salud bucal (Wamala et al., 2006) y consultan menos al dentista, incluso contando con el beneficio de algunos seguros (Bedos et al., 2009). En Chile, de la población que percibe la necesidad de atención dental, un $22,6 \%$ solicita atención y finalmente, solo $20,6 \%$ la recibe, existiendo una gradiente de inequidad según NSE, en relación a recibir atención dental. Otro aspecto importante es que las personas afiliadas a las Instituciones de salud previsional (Isapre) demandan atención de salud dental en mayor proporción que los afiliados al Fondo Nacional de Salud (FONASA), observándose también una gradiente de inequidad por NSE (Delgado et al., 2013). La diferencia de demanda de atención según NSE se explica porque las personas con menos recursos, que perciben que los servicios no son suficientes, no demandan la atención (Frenk, 1997).

Una de las estrategias implementadas en nuestro país para disminuir inequidades en salud bucal y responder a la alta necesidad de tratamiento es la atención odontológica integral de la embarazada, garantizada por el régimen de Garantías Explícitas en Salud (GES), que está dirigida a educar, prevenir, recuperar y rehabilitar la salud bucal de la madre, ya que su estado de salud bucal influirá en el riesgo de desarrollar caries tempranas en los niños, a través de mecanismos biológicos, conductuales y sociales. (Zanata et al., 2003; Mofidi et al,. 2009).

Se ha descrito que existe un mayor riesgo de presentar caries y gingivitis durante el embarazo (Rakchanok et al., 2010), ya que se modifican las conductas relacionadas con la salud bucal, teniendo como consecuencia el aumento de la acidez en la cavidad oral, debido al mayor consumo de azúcar asociado a los "antojos" y a la menor atención a los cuidados de salud bucal (Silk et al., 2008). Las alteraciones fisiológicas propias de este periodo incluyen una inmunosupresión temporal y el aumento de niveles hormonales de estrógeno y progesterona (Pramanik et al., 2007), las que sirven de nutrientes para las bacterias patógenas periodontales, aumentando la carga bacteriana total, dando como resultado una mayor severidad de los signos y síntomas a nivel periodontal (Raber et al., 1994).

La evidencia internacional revela que las embarazadas no acuden al dentista ( Honkala \& Al-Ansari, 2005; Detman et al,. 2010; Keirse \& Plutzer, 2010; Hunter \& Yount, 2011) o solo van cuando presentan dolor (Hashim, 2012). En Chile, solo el 31\% de mujeres embarazadas atendidas en los servicios públicos y privados recibieron atención odontológica (Núñez et al., 2013), y datos del Departamento de Estadística del Ministerio de Salud (DEIS) indican que el $14 \%$ de las mujeres embarazadas que ingresan a tratamiento odontológico en Atención Primaria de Salud (APS) lo abandonan antes del alta (DEIS, 2013).

Esta realidad hace necesario conocer cuáles son las barreras de acceso a la atención odontológica para las embarazadas, tomar medidas conducentes a mejorar su estado de salud bucal e impactar en la salud bucal de sus hijos (Zanata et al., 2003; Mofidi et al, 2009). Se debe tener en cuenta que para muchas mujeres, el embarazo es una oportunidad única para modificar conductas asociadas a un riesgo mayor de problemas de salud bucal y general (MINSAL, 2013).

\section{Metodología}

La presente revisión narrativa tiene por objetivo revisar la evidencia internacional y nacional respecto a las barreras que impiden el acceso a la atención odontológica de las mujeres embarazadas.

Se realizó una búsqueda amplia en las bases de datos Pubmed y Bireme, en el metabuscador Epistemonikos y buscador Google con los términos "embarazo", "cuidado dental"," "acceso a los servicios de salud", "barreras para la atención"," cobertura de atención" $y$ “GES". Los criterios de inclusión fueron: artículos relacionados con el tema de estudio (barreras para la atención odontológica de embarazadas) e idioma de publicación español, inglés y portugués, no se limitó por año del artículo ni por tipo de estudio (cualitativo, cuantitativo). Una vez eliminados los duplicados, se obtuvieron 244 artículos y documentos, de los cuales se seleccionaron 16 que cumplían con los criterios de inclusión antes mencionados.

\section{Resultados}

\section{1.- Evidencia internacional}

La literatura internacional ha descrito barreras para el cuidado de salud bucal en las embarazadas, las que se han clasificado en: 1) Barreras estructurales, que son las relacionadas con la organización 
del sistema de salud o de los proveedores de las atenciones de salud y 2) Barreras personales de las mujeres y sus creencias (Association \& American College of Obstetricians and Gynecologists 2010).

\section{1.- Barreras estructurales}

\section{Costo y acceso}

Diferentes autores han reportado que los altos costos, la baja cobertura de algunos seguros (bajos reembolsos) o la ausencia de estos representan una barrera para que las mujeres embarazadas accedan a la atención odontológica (Boggess \& Edelstein, 2006; California Dental Association Foundation, \& American College of Obstetricians and Gynecologists, District IX. 2010; Marchi et al., 2010; Bahadori et al., 2013).

Se ha descrito que la mayor barrera es el acceso a los prestadores de atención (Bedos et al., 2009; Detman et al., 2010; Farias Souza \& Lima Chaves, 2010; Corchuelo, 2013; Quintero et al., 2014). Estos problemas de acceso se relacionan con la falta de recursos económicos para costear la atención odontológica, la distancia hasta el centro de salud y la disponibilidad de profesionales dispuestos a realizar la atención en mujeres embarazadas (Bahadori et al., 2013; Amin \& ElSalhy, 2014).

\section{Características de los proveedores de la atención de salud}

Las creencias de los odontólogos se han descrito como una barrera para el acceso de las atenciones de salud de las embarazadas. Esto debido al miedo de los profesionales a demandas judiciales derivados de posibles complicaciones en la atención, a las percepción de falta de competencias para enfrentar la atención de las embarazadas o por las dificultades propias de su atención (Le et al., 2009; California Dental Association Foundation, \& American College of Obstetricians and Gynecologists, District IX. 2010; Bahadori et al., 2013). La falta de competencias de los odontólogos es una barrera para el acceso y se describe en la literatura que se relaciona con falencias en las habilidades de comunicación y no con la falta de habilidades clínicas (Le et al., 2009). Finalmente, en relación con las características de los odontólogos, las embarazadas relatan una relación insatisfactoria debido a actitudes tales como dentistas que no responden a sus preguntas o que no dan suficiente información (Bahadori et al., 2013).

\section{2.- Barreras personales}

\section{Percepción de necesidad de tratamiento}

Gran cantidad de mujeres no perciben la necesidad de recibir tratamiento dental durante el embarazo, esto debido a la creencia de que los problemas dentales son normales durante este periodo y no requieren de cuidados especiales (Albuquerque et al., 2004; California Dental Association Foundation, \& American College of Obstetricians and Gynecologists, District IX. 2010; Keirse \& Plutzer, 2010; Marchi et al., 2010; Amin \& ElSalhy, 2014).

\section{Miedo a la atención}

La atención odontológica durante el embarazo produce miedo, esto debido a la creencia errónea de que no es seguro para el feto o por miedo al dolor asociado a la atención odontológica (Albuquerque et al., 2004; Le et al., 2009; California Dental Association Foundation, \& American College of Obstetricians and Gynecologists, District IX.2010; Hunter and Yount, 2011; Bahadori et al., 2013).

\section{Valoración de la salud bucal}

La falta de comprensión de la importancia de la salud bucal en general y durante el embarazo también se ha descrito como una barrera, haciendo que la atención odontológica durante este periodo no sea considerada como una prioridad (Boggess \& Edelstein, 2006; Le et al., 2009; Hunter \& Yount, 2011).

\section{2.- Panorama Nacional}

Desde julio 2010, el Estado de Chile garantiza a las mujeres embarazadas el acceso, calidad, oportunidad y protección financiera para la atención integral de su salud bucal, a través del AUGE (MINSAL, 2016). Sin embargo, a pesar de ser una condición garantizada, esto no se traduce en una mayor cantidad de ingresos a tratamiento y altas por término de este (DEIS, 2013).

Algunos autores han sugerido que la baja utilización de las prestaciones garantizadas del GES puede deberse, entre otros factores, al hecho de ser atención ambulatoria y al desconocimiento de la cobertura (Fernández , 2007). En Chile, no existen estudios que exploren las barreras para el acceso a la atención odontológica de mujeres embarazadas. Si bien es cierto, estos problemas se pueden atribuir a barreras descritas en la literatura, existen diferencias culturales y propias de la organización del sistema de salud local que se deben tener en cuenta.

\section{Conclusiones}

La evidencia internacional describe barreras de acceso a la atención odontológica de las mujeres embarazadas, estas se pueden dividir en barreras estructurales, tales como: costo de la atención, acceso a los servicios y características de los proveedores de la atención de salud, y las barreras personales, que incluyen: la percepción de necesidad de tratamiento, el miedo a la atención y la poca valoración de la salud bucal. Se hace necesario, entonces, realizar estudios nacionales que exploren las barreras de acceso a la atención de las embarazadas chilenas en el GES. Se sugiere especialmente utilizar la metodología cualitativa, ya que permitirá comprender el fenómeno en profundidad, debido a las diferencias del contexto local, lo que permitirá diseñar estrategias orientadas a enfrentarlas y mejorar el sistema de atención. 


\section{Referencias}

Amin M \& ElSalhy, M. (2014). Factors Affecting Utilization of Dental Services During Pregnancy. Journal of Periodontology, 85, 1712-1721.

Albuquerque O. M. R. de, Abegg, C \& Rodrigues C. S. (2004). Percepção de gestantes do Programa Saúde da Família em relação a barreiras no atendimento odontológico em Pernambuco, Brasil. Cadernos de Saúde Pública, 20, 789-796.

Bahadori, M, Ravangard, R \& Asghari B. (2013). Perceived Barriers Affecting Access to Preventive Dental Services: Application of DEMATEL Method. Iranian Red Crescent Medical Journal, 15, 655-662.

Bedos, C, Levine, A \& Brodeur J.M. (2009). How People on Social Assistance Perceive, Experience, and Improve Oral Health. Journal of Dental Research, 88, 653-657.

Boggess K. A \& Edelstein B. L. (2006). Oral health in women during preconception and pregnancy: implications for birth outcomes and infant oral health. Maternal and Child Health Journal, 10 (5 Suppl), 169-174.

Corchuelo, J. (2013). Determinantes sociales y del estilo de vida en salud oral en el acceso a odontología de gestantes caleñas en el 2012. Recuperado de: http://www.redalyc.org/articulo. oa?id=12028727017

California Dental Association Foundation, \& American College of Obstetricians and Gynecologists, District IX. (2010). Oral health during pregnancy and early childhood: evidence-based guidelines for health professionals. Journal of the California Dental Association, 38, 391-403, 405-440.

DEIS (2013). Base de datos REM A09 sección C. REM 2013. Disponible en http://www.deis.cl/rem-2013-2/

Delgado I, Cornejo M, Jadue L \& Huberman J. (2013). Determinantes sociales y equidad de acceso en la salud dental en Chile', CIENT.DENT 10, 15-23.

Detman L, Cottrell B \& Denis-Luque M. (2010). Exploring Dental Care Misconceptions and Barriers in Pregnancy. Birth 37, 318-324.

Farias Souza L \& Lima Chaves S. C. (2010). Política nacional de saúde bucal: Acessibilidade e utilização de serviços odontológicos especializados em um município de médio porte na Bahía. Revista Baiana de Saúde Pública. 34, 371-387.

Fernández R. (2007). ¿GES O NO GES? Esa es la cuestión. Rev Esp Econ Salud, 5, 361-368.
Frenk J. (1997). La salud de la población. Hacia una nueva salud pública. Fondo de cultura económica México. Disponible en: http://bibliotecadigital.ilce.edu.mx/sites/ciencia/volumen3/ciencia3/133/htm/poblacion.htm

Hashim R. (2012). Self-reported oral health, oral hygiene habits and dental service utilization among pregnant women in United Arab Emirates. International Journal of Dental Hygiene, 10, 142-146.

Honkala S \& Al-Ansari J. (2005). Self-reported oral health, oral hygiene habits, and dental attendance of pregnant women in Kuwait. Journal of Clinical Periodontology, 32, 809-814.

Hunter L \& Yount S. (2011). Oral health and oral health care practices among low-income pregnant women. Journal of midwifery \& women's health, 56, 103-9.

Keirse MJ \& Plutzer K. (2010). Women's attitudes to and perceptions of oral health and dental care during pregnancy. Journal of Perinatal Medicine. 38, 3-8.

Le M, Riedy C, Weinstein P \& Milgrom P. (2009). Barriers to Utilization of Dental Services during Pregnancy: A Qualitative Analysis. J Dent Child 76, 46-52.

Marchi K, Fisher-Owen S, Weintraub J, Yu Z. \& Braveman P. (2010). Most pregnant women in California do not receive dental care: findings from a population-based study. Public health reports 125, 831-842.

MINSAL (2003). Encuesta nacional de salud ENS 2003, ed. Departamento de Salud Pública UC. Disponible en: http://web.minsal. cl/portal/url/item/bcb03d7bc28b64dfe040010165012d23.pdf

MINSAL (2010). Análisis de situación de salud bucal en Chile. Disponible en: http://web.minsal.cl/sites/default/files/files/Análisis\%20 de\%20Situación\%20Salud\%20Bucal\%20final\%20pdf.pdf

MINSAL (2013). Guía Clínica AUGE Salud Oral Integral de la Embarazada. Disponible en: http://www.supersalud.gob.cl/difusion/572/ articles-6455_recurso_1.pdf

MINSAL (2016). Orientación en Salud. Superintendencia de Salud, Gobierno de Chile. Salud oral integral de la embarazada. Disponible en http://www.supersalud.gob.cl/difusion/665/ w3-article-5997.html

Mofidi M, Zeldin L \& Rozier R. (2009). Oral Health of Early Head Start Children : A Qualitative Study of Staff, Parents and Pregnant Women. American Journal of Public Health 99, 245-252. 


\section{Cantarutti et al.}

Núñez J, Moya P, Monsalves M. J \& Landaeta M S. (2013). Nivel de Conocimiento de Salud Oral y Utilización de GES Odontológico en Puérperas Atendidas en una Clínica Privada, Santiago, Chile. International Journal of Odontostomatology, 7, 39-46.

OMS (2012). Salud bucodental, Nota informativa N³18. Disponible en: http://www.who.int/mediacentre/factsheets/fs318/es/

Pramanik S, Pramanik T, Mondal S.C \& Chanda R. (2007). Number, maturity and phagocytic activity of neutrophils in the three trimesters of pregnancy. Eastern Mediterranean Health Journal. 13, 862-867.

Quintero C, Robledo D, Vásquez A, Delgado O \& Franco A. (2014). Barreras de acceso a la atención odontológica durante la primera infancia Medellín 2007. Rev Fac Odontol Univ Antioq, 25, 325-341.

Raber-Durlacher J, van Steenbergen T, Van der Velden U, de Graaff J \& Abraham-Inpijn L. (1994). Experimental gingivitis during pregnancy and post-partum: clinical, endocrinological, and microbiological aspects. J. Clin. Periodontol, 21, 549-558.
Rakchanok N, Amporn D, Yoshida Y, Harun-Or-Rashid M, Sakamoto J. (2010). Dental caries and gingivitis among pregnant and non-pregnant women in Chiang Mai, Thailand. Nagoya J Med Sci. 72, 43-50.

Silk H, Douglass D, Douglass J \& Silk L. (2008) 'Oral health during pregnancy. American Family Physician 77, 1139-1144

Wamala, S, Merlo J \& Boström G. (2006). Inequity in access to dental care services explains current socioeconomic disparities in oral health: the Swedish National Surveys of Public Health 2004-2005. Journal of Epidemiology and Community Health, 60, 1027-1033.

Zanata R. L, Navarro M. F. de L, Pereira J. C, Franco E. B, Lauris, J. R. P \& Barbosa S. H. (2003). Effect of caries preventive measures directed to expectant mothers on caries experience in their children. Brazilian Dental Journal, 14, 75-81. 